\title{
Treatment of upper urinary tract stones with flexible ureteroscopy in children
}

Jing Xiao; Xiangyu Wang; Jun Li; Miaoiao Wang; Tiandong Han; Caixiang Zhang; Yuan Du; Gangyue Hao; Ye Tian

Department of Urology, Beijing Friendship Hospital, Capital Medical University, Beijing, China

Acknowledgments: This work was supported by the Beijing Municipal Science \& Technology Commission (Z151100004015106) and the Beijing Municipal Administration of Hospital`s Youth Program (QML20150103).

Cite as: Can Urol Assoc $J 2018$ August 30; Epub ahead of print. http://dx.doi.org/10.5489/cuaj.5283

Published online August 30, 2018

$* * *$

\section{Abstract}

Introduction: This single-centre, retrospective study aimed to assess the efficacy and safety of flexible ureteroscopy (FURS) combined with holmium laser lithotripsy in treating children with upper urinary tract stones.

Methods: From June 2014 to October 2015, a total of 100 children (74 boys and 26 girls) with upper urinary tract stones were treated using FURS. A 4.7 Fr Double-J stent was placed two weeks before operation. Patients were considered stone-free when the absence of residual fragments was observed on imaging studies. The preoperative, operative, and postoperative data of the patients were retrospectively analyzed.

Results: A total of 100 pediatric patients with a mean age of $3.51 \pm 1.82$ years underwent 131 FURS and holmium laser lithotripsy. Mean stone diameter was $1.49 \pm 0.92 \mathrm{~cm}$. Average operation time was 30.8 minutes (range 15-60). The laser power was controlled between 18 and $32 \mathrm{~W}$, and the energy maintained between 0.6 and $0.8 \mathrm{~J}$ at any time; laser frequency was controlled between 30 and $40 \mathrm{~Hz}$. Complications were observed in 69 (69.0\%) patients and classified according to the Clavien system. Postoperative hematuria (Clavien I) occurred in 64 (64.0 \%) patients. Postoperative urinary tract infection with fever (Clavien II) was observed in eight (7\%) patients. No ureteral perforation and mucosa avulsion occurred. The overall stone-free rate of single operation was 89/100(89\%). Stone diameter and staghorn calculi were significantly associated with stone-free rate.

Conclusions: FURS and holmium laser lithotripsy is effective and safe in treating children with upper urinary tract stones. 


\section{Introduction}

Urinary stone disease (USD) remains a clinical challenge. Indeed, about 1 in 11 individuals in the United States are affected by kidney stones in their lifetime; ${ }^{1}$ with a prevalence similar to that of diabetes, USD represents one of the most expensive urologic conditions, with health care charges exceeding $\$ 10$ billion annually. ${ }^{2}$ Unfortunately, its incidence has increased among young patients, particularly women and blacks in the past years. ${ }^{3,4}$ Treatment of children with urinary stones has always been a challenge for urologists. ${ }^{5}$ The current treatment for pediatric patients with urinary calculi is based on adult procedures, including extracorporeal shock wave lithotripsy (ESWL), percutaneous nephrolithotomy (PCNL), ureteroscopy lithotripsy (URL), and laparoscopic ureterolithotomy ${ }^{6-9}$ Due to their particular physiological characteristics, surgical intervention method for pediatric nephrolithiasis should be approached differently compared to adults.

Flexible ureteroscopy (FURS) has been applied in recent years to the treatment of urinary stones, which presents overt advantages over other techniques, including improved resolution and increased optical field, high stone-free rate, reduced risk of bleeding, limited surgical injury, good repeatability, and speedy recovery; in addition, further miniaturization is possible. ${ }^{10-12}$ In children and infants, who have a relatively high stone recurrence rate, ${ }^{13}$ flexible ureteroscopy could be performed repeatedly. FURS and holmium laser lithotripsy in treating infants and children has been previously assessed. ${ }^{14-16}$ However, most reports were limited in sample size.

\section{Methods}

This was a retrospective study of one hundred children (74 boys and 26 girls) who underwent FURS and holmium laser lithotripsy for upper urinary tract stones between June 2014 and October 2015 at our hospital, Beijing Friendship Hospital.

The selection criteria for the procedure included SWL-refractory stones, upon parent's and/or surgeon's preference. Preoperative imaging scans, including a plain abdominal radiograph (KUB), urinary ultrasound (USG), low-dose non-contrast computerized tomography (NCCT) and/or intravenous urogram (IVP) were obtained from all patients. The stone size was taken as the longest diameter measured on CT or cumulative stone diameter defined as the sum of longest diameter of each stone. A urine sample was obtained for routine urinalysis and bacterial culture before the operation. An indwelling 4.7 Fr double J-stent (Bloomington, COOK Company, USA) was placed for 2 weeks in advance, with the length derived as age $+10(\mathrm{~cm})$, according to a previous report. ${ }^{17}$

The operations were completed by surgeon JL, a urologist with more than 10 years experience in treating urolithiasis. After induction of general anesthesia using a laryngeal mask for airway management, prophylactic intravenous antibiotics (200 $\mathrm{mg} / \mathrm{kg} / \mathrm{d}$ fosfomycin dissolved in $5 \%$ glucose solution or glucose-sodium chloride solution) were administered. Then, a pediatric forced-air warming blanket (Figure 1A) 
was placed, with the surgical field disinfected with iodine pre-warmed to $36^{\circ} \mathrm{C}$. The double J-stent was removed with an $8.0 / 9.8 \mathrm{Fr}$ or $4.5 / 6.5 \mathrm{Fr}$ rigid ureteroscope (WOLF Company, Germany). A $14 \mathrm{G}$ trocar (outer diameter $2.108 \mathrm{~mm}$ ) was used to perform a suprapubic puncture cystostomy to allow fluid drainage from the bladder during operation(Figure 1B), which was first introduced for such operations.

The ureter was explored through an 8.0/9.8Fr or 4.5/6.5Fr ureteroscope, and stones located in the ureteropelvic junction were pushed into the renal pelvis softly. For stones located in other places, FURL was performed directly if the ureter was not narrow or circuitous. A POLY flexible ureteroscope (PolyDiagnost, Germany, Figure 1C) was placed into the target ureter or renal pelvis alongside the ureteral access sheath (UAS) (9.5/11.5 Fr). All stones were thoroughly smashed, and the fragments were flushed into the renal pelvis. A $200-\mu \mathrm{m}$ holmium laser optical fiber was connected to the ureteroscope to perform lithotripsy. The stones in lower calyx were moved by the nickel-titanium reticular basket before laser lithotripsy (Figure 1D). Laser frequency was 30-40 Hz and energy was 0.6-0.8 J. Low-energy laser was used for lithotripsy, which could shatter the stones into powder, promote the excretion of stone debris, and reduce the incidence of "stone street" after the operation. The laser power could be increased to $0.8 \mathrm{~J}$ for cystine stones. During surgery, perfusion pressure of the irrigating fluid did not exceed $40 \mathrm{cmH}_{2} \mathrm{O}$, with irrigating fluid volume $\leq 1,000 \mathrm{ml}$.

All children underwent abdominal radiography on the first postoperative day to confirm the position of the double J-stent. The double J stent was removed 4 weeks after operation if retained intraoperatively. Kidney ultrasound and KUB were reexamined every 3 months in the first year, and every six months the following years. Clinically insignificant residual fragments (CIRFs) were recorded. ${ }^{18}$

Patients were considered stone-free if residual fragments $<4 \mathrm{~mm}$ were observed on imaging studies, which were performed at 1 month following the operation. If residual calculi $>4 \mathrm{~mm}$ were observed, second-stage RIRS was performed. Patients with residual calculi $<4 \mathrm{~mm}$ continued to the follow-up.

General information, perioperative signs and symptoms, laboratory data, respective examination results, operation safety indicators such as operation time, laser power, perfusion volume, and postoperative follow-up data were collected for all patients.

Quantitative data were expressed as mean \pm standard deviation (SD). Multivariate logistic regression was performed to identify the factors associated with stone free status. Data were analyzed with SPSS ver. 17.0 (IBM Co., Armonk, NY, USA). $\mathrm{P}<0.05$ was considered statistically significant.

\section{Results}

There were 25, 45, and 30 cases of ureteral-, kidney-, and co-existing renal and ureteral calculi, respectively. The mean age of the patients was $3.51 \pm 1.82$ years, ranging from 4 to 168 months; mean stone diameter was $1.49 \pm 0.92 \mathrm{~cm}$, ranging 
between 0.5 and $3 \mathrm{~cm}$. Table 1 shows the patient and stone characteristics.

A total of 100 pediatric patients underwent 131 FURS and holmium laser lithotripsy. 19 patients had bilateral stones, and we recorded it as 2 FURS if the patient had lithotripsy on both sides in stage I. 12 patients required repeat operations and only 1 needed a third operation. Average operation time was $30.8 \mathrm{~min}$ (15 to 60 $\mathrm{min}$ ), with a mean volume of perfusate of $442.7 \mathrm{ml}$ (200-1000 ml). The laser power was controlled between 18 and 32W, and the energy maintained between 0.6 and $0.8 \mathrm{~J}$ at any time; laser frequency was controlled between 30 and $40 \mathrm{~Hz}$. Complications were observed in $69(69.0 \%)$ patients and classified according to the Clavien system. Postoperative hematuria (Clavien I) occurred in 64 (64.0\%)patients and was resolved with hydration. Post-operative urinary tract infection with fever (Clavien II) was observed in 8/113 (7\%) patients and treated with antibiotics. No ureteral perforation and mucosa avulsion occurred.(Table 2).

The overall stone free rate of single operation was 89/100(89\%).Several factors were assessed for their associations with stone free rate, including age, gender, stone diameter, stone location, stag-horn calculi, and hydronephrosis. Only stone diameter and stag-horn calculi were significantly associated with stone free rate; these two variables were confirmed as significant risk factors for stone free rate (stone diameter, OR=3.274 95\%CI 1.124-9.533, $\mathrm{P}=0.03$; stag-horn calculi, $\mathrm{OR}=13.193$ 95\%CI 1.881-92.537, $\mathrm{P}=0.009$ ) (Table 3).

Stone analysis was available in 65 patients. The stone composition was calcium oxalate in 38 patients, cystine in 10 patients and magnesiumammonium phosphate in 17 (17\%) patients. Two children were diagnosed primary hyperoxaluria.

\section{Discussion}

The standard procedures to treat stones in children are similar to those used in adults. ${ }^{6-9}$ A prospective study of 60 pre-school children demonstrated that FURS with holmium laser lithotripsy is as safe as ESWL, but higher stone-free rate (86.6\% vs. $70.0 \%) .{ }^{19}$ In a recent study, 100 SWL and 46 F-URS were conducted in 69 children, The SF rate after one procedure was almost two times higher in the F-URS group compared with the SWL group (37\% vs $21 \% ; p=0.04)$ without increasing the complication rate $(21.7 \%$ vs $16 \% ; p=0.31) .{ }^{20}$ For large or complex renal stones in pediatric patients, percutaneous nephrolithotomy monotherapy has the advantage of improved stone-free rates, while retrograde intrarenal surgery decreases radiation exposure, complications and hospital stay. ${ }^{21}$

As in adults, PCNL is recommended as a first-line treatment of choice for renal stones larger than $2 \mathrm{~cm}$ in children. ${ }^{22}$ In children with urinary calculi, kidney volume is only about half that of an adult, making puncture more challenging with increased risk of hemorrhage and other complications. The development of scar tissues after PCNL ${ }^{23}$ may also affect development of the infant kidney. Also, many infant urinary calculi are caused by metabolic disturbance and are therefore likely to recur. ${ }^{24-26}$ In this study, we found 2 children had primary hyperoxaluria, an autosomal 
recessive disease, and 10 children presented with cystine calculi. These children may need more than one operation in their lifetime.

Although 50 years have elapsed since Marshall first described the inspection of a ureteral calculus with a flexible ureteroscope, the wide application of this technique in the diagnosis and treatment of upper urinary tract disease is relatively recent. ${ }^{27}$ Flexible ureteroscopy is considered the first choice for children with lower calyceal stones $<15 \mathrm{~mm}$ in diameter. ${ }^{28}$ Galal and colleagues reported that the main FURS complications in children with ureteral calculi were clinically insignificant hematuria, renal colic and fever. ${ }^{29}$ Jurkiewicz and colleagues reported a stone-free rate of $85.3 \%$ in a cohort of 157 juveniles (10 months to 17 years) using FURS and holmium laser lithotripsy. ${ }^{30}$

Stone size is a critical factor influencing the primary stone-free rate. The largest and smallest stones in the current cohort measured $3.5 \times 2.5 \mathrm{~cm}$ and $0.4 \times 0.5$ $\mathrm{cm}$, respectively, and an overall stone-free rate of $89 \%$ was obtained. Stone diameter is a significant risk factor for stone free rate. The Children's Hospital of Philadelphia (USA) reported stone-free rates of $100 \%$ and $97 \%$ for individuals with stones $<10 \mathrm{~mm}$ and $\geq 10 \mathrm{~mm}$ in diameter, respectively; analysis of recurrence and complications over a median follow-up of 19.7 months suggested that FURS is a safe and effective means of treating upper ureteral stones and stones in the lower renal calyx. ${ }^{17}$ Unsal and colleagues reported a stone-free rate of $100 \%$ for stones $<10 \mathrm{~mm}$ diameter and $81.8 \%$ for larger stones in a group of 16 children who underwent FURS and holmium laser lithotripsy. ${ }^{14}$ Jurkiewicz and colleagues reported a stone-free rate of $85.3 \%$ in their cohort of 157 juveniles (aged between 10 months and 17 years) using FURS and holmium laser lithotripsy: primary stone-free rate reached $85.3 \%$, and total stone-free rate was $98.1 \%$; only 3 patients had complications. ${ }^{30}$ Furthermore, Chedgy assessed 21 patients, and $13(62 \%)$ were stone free after the first procedure, 17 (81\%) after a second one, and 20 (95\%) after a third operation. ${ }^{15}$ Ishii ${ }^{16}$ carried out a meta-analysis of 6 studies including 282 cases of juveniles (0.25 -17 years) that underwent FURS and holmium laser lithotripsy: primary stone-free rate in 3 articles was 85.8\% (58.0$93.0 \%$ ) while total complication rate was $12.4 \%$; only 5 cases had Clavien class III complications. Taken together, these findings demonstrated that FURS and holmium laser lithotripsy conducted is safe and effective in pediatric patients with stone dimeter around $1 \mathrm{~cm}$.

In our study, we do had a double J-stent placed in advance. This made UAS placement successful in all patient. According to previous study, a UAS placement could possible in $94.1 \%$ of the pre-stented patients, but only in $50 \%$ of the nonstented patients. ${ }^{31}$ The use of a UAS during RIRS has been associated with reduced intrarenal pressure, decreased operative time and improved SFR in adult patients. ${ }^{32}$ So, we placed a double J-stent in all patients in advance. No ureteral wall injury occurred in our patients.

The limiting pressure within the urinary tract is also critical: the outflow must keep unobstructed. Suprapubic puncture cystostomy may be required to achieve constant 
drainage. Close attention should be paid to preventing intraoperative hypothermia. We recommended using pre-warming irrigation, a forced-air warming blanket, insulating the head and maintaining a relatively high operating room temperature. Finally, we recommend that laser power be limited to $32 \mathrm{~W}$ to avoid mucosal damage while disrupting calculi. It is better to modulate the laser with high-frequency and lowpower.

A few limitations should be mentioned for this study. It was a single-center retrospective cohort study with a relatively short follow-up time. Also, a control group of patients treated with PCNL or ESWL was not included. The suprapubic puncture cystotomy was for decompression of the bladder. But more research should do to prove that this procedure decrease the complication rates. Further RCT studies are warranted to compare the two methods for advantages and disadvantages.

Generally speaking, with sufficient preoperative preparation and skilled operative manipulation, FURS and holmium laser lithotripsy has a high stone-free rate (SFR) and clinical safety for upper urinary tract stones in children. And our study is one of the largest series in the literature to show the safety and efficacy of RIRS in children. 


\section{References}

1. C. D. Scales, Jr., A. C. Smith, J. M. Hanley, et al., Prevalence of kidney stones in the united states, Eur Urol, 62 (2012), 160-5.

2. C. D. Scales, Jr., G. E. Tasian, A. L. Schwaderer, et al., Urinary stone disease: Advancing knowledge, patient care, and population health, Clin J Am Soc Nephrol (2016).

3. A. Hesse, Reliable data from diverse regions of the world exist to show that there has been a steady increase in the prevalence of urolithiasis, World J Urol, 23 (2005), 302-3.

4. G. E. Tasian, M. E. Ross, L. Song, et al., Annual incidence of nephrolithiasis among children and adults in south carolina from 1997 to 2012, Clin J Am Soc Nephrol, 11 (2016), 488-96.

5. E. Minevich, and C. A. Sheldon, The role of ureteroscopy in pediatric urology, Curr Opin Urol, 16 (2006), 295-8.

6. H. Sen, I. Seckiner, O. Bayrak, et al., Treatment alternatives for urinary system stone disease in preschool aged children: Results of 616 cases, J Pediatr Urol, 11 (2015), 34 e1-5.

7. H. Ishii, S. Griffin, and B. K. Somani, Ureteroscopy for stone disease in the paediatric population: A systematic review, BJU Int, 115 (2015), 867-73.

8. J. C. Thomas, How effective is ureteroscopy in the treatment of pediatric stone disease?, Urol Res, 38 (2010), 333-5.

9. M. C. Smaldone, S. G. Docimo, and M. C. Ost, Contemporary surgical management of pediatric urolithiasis, Urol Clin North Am, 37 (2010), 253-67.

10. C. Almeras, M. Daudon, G. Ploussard, et al., Endoscopic description of renal papillary abnormalities in stone disease by flexible ureteroscopy: A proposed classification of severity and type, World J Urol (2016).

11. X. Zhu, L. Song, D. Xie, et al., Animal experimental study to test application of intelligent pressure control device in monitoring and control of renal pelvic pressure during flexible ureteroscopy, Urology, 91 (2016), 242 e11-5.

12. J. Rassweiler, M. C. Rassweiler, and J. Klein, New technology in ureteroscopy and percutaneous nephrolithotomy, Curr Opin Urol, 26 (2016), 95-106.

13. I. Oral, I. Nalbant, U. Ozturk, et al., Our experience with percutaneous nephrolithotomy in pediatric renal stone disease, Turk J Urol, 39 (2013), 35-8.

14. A. Unsal, and B. Resorlu, Retrograde intrarenal surgery in infants and preschool-age children, J Pediatr Surg, 46 (2011), 2195-9.

15. E. C. Chedgy, S. J. Griffin, J. P. Dyer, et al., Ureteroscopy for paediatric renal tract stones - outcomes from a tertiary european centre, Urol Int, 95 (2015), 320-3.

16. H. Ishii, S. Griffin, and B. K. Somani, Flexible ureteroscopy and lasertripsy (fursl) for paediatric renal calculi: Results from a systematic review, J Pediatr Urol, 10 (2014), 1020-5.

17. S. S. Kim, T. F. Kolon, D. Canter, et al., Pediatric flexible ureteroscopic 
lithotripsy: The children's hospital of philadelphia experience, J Urol, 180 (2008), 2616-9; discussion 19.

18. D. G. Assimos, Re: Clinically insignificant residual fragments: Is it an appropriate term in children?, J Urol, 195 (2016), 170.

19. I. A. Mokhless, H. M. Abdeldaeim, A. Saad, et al., Retrograde intrarenal surgery monotherapy versus shock wave lithotripsy for stones 10 to $20 \mathrm{~mm}$ in preschool children: A prospective, randomized study, J Urol, 191 (2014), 1496-9.

20. L. Freton, B. Peyronnet, A. Arnaud, et al., Extracorporeal shockwave lithotripsy versus flexible ureteroscopy for the management of upper tract urinary stones in children, J Endourol, 31 (2017), 1-6.

21. K. S. Saad, M. E. Youssif, S. Al Islam Nafis Hamdy, et al., Percutaneous nephrolithotomy vs retrograde intrarenal surgery for large renal stones in pediatric patients: A randomized controlled trial, J Urol, 194 (2015), 1716-20.

22. C. Turk, A. Petrik, K. Sarica, et al., Eau guidelines on interventional treatment for urolithiasis, Eur Urol, 69 (2016), 475-82.

23. K. Wood, T. Keys, P. Mufarrij, et al., Impact of stone removal on renal function: A review, Rev Urol, 13 (2011), 73-89.

24. L. Copelovitch, Urolithiasis in children: Medical approach, Pediatr Clin North Am, 59 (2012), 881-96.

25. C. F. Granberg, and L. A. Baker, Urolithiasis in children: Surgical approach, Pediatr Clin North Am, 59 (2012), 897-908.

26. H. Alpay, A. Ozen, I. Gokce, et al., Clinical and metabolic features of urolithiasis and microlithiasis in children, Pediatr Nephrol, 24 (2009), 2203-9.

27. V. F. Marshall, Fiber optics in urology, J Urol, 91 (1964), 110-4.

28. I. Gecit, N. Pirincci, M. Gunes, et al., Should ureteroscopy be considered as the first choice for proximal ureter stones of children?, Eur Rev Med Pharmacol Sci, 17 (2013), 1839-44.

29. E. M. Galal, T. K. Fath El-Bab, and A. M. Abdelhamid, Outcome of ureteroscopy for treatment of pediatric ureteral stones, J Pediatr Urol, 9 (2013), 476-8.

30. B. Jurkiewicz, T. Zabkowski, and J. Samotyjek, Ureterolithotripsy in a paediatric population: A single institution's experience, Urolithiasis, 42 (2014), 171-6.

31. B. Erkurt, T. Caskurlu, G. Atis, et al., Treatment of renal stones with flexible ureteroscopy in preschool age children, Urolithiasis, 42 (2014), 241-5.

32. O. L'Esperance J, W. O. Ekeruo, C. D. Scales, Jr., et al., Effect of ureteral access sheath on stone-free rates in patients undergoing ureteroscopic management of renal calculi, Urology, 66 (2005), 252-5. 


\section{Figures and Tables}

Fig. 1. Surgical procedure and key materials.
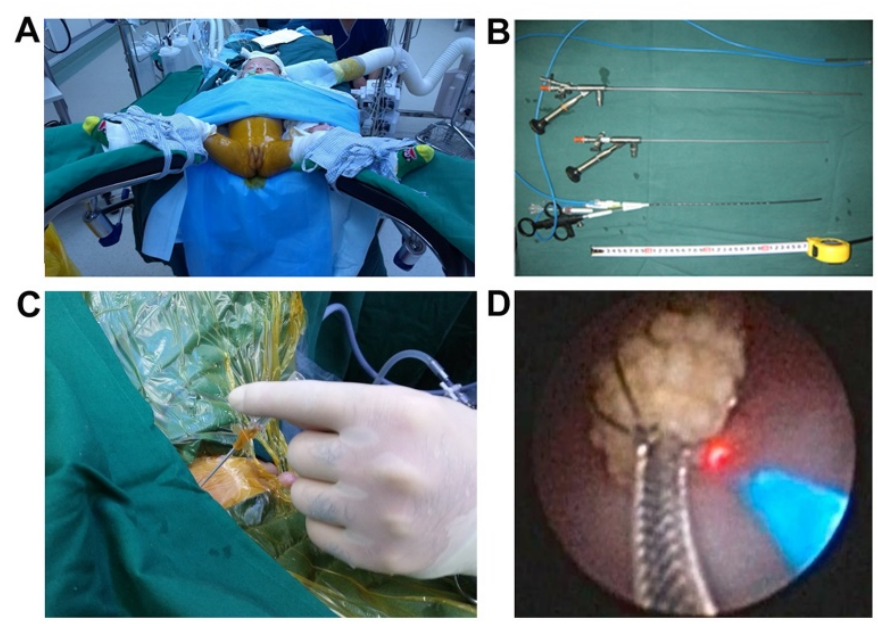

\begin{tabular}{|l|c|}
\hline \multicolumn{2}{|l|}{ Table 1. Baseline characteristics of patients and stone } \\
\hline Mean age (years) & $3.51 \pm 1.82(4-168$ months) \\
\hline Male/female & $74 / 26$ \\
\hline Stone diameter (cm) & $1.49 \pm 0.92(0.5-3)$ \\
\hline Lateralization (L/R/B) & $42 / 39 / 19$ \\
\hline Stone location & 23 \\
\hline Renal pelvis & 11 \\
\hline Upper pole calyx & 13 \\
\hline Mid pole calyx & 28 \\
\hline Lower pole calyx & 55 \\
\hline Ureter & 26 \\
\hline Symptom & 26 \\
\hline Back pain & 8 \\
\hline Hematuria & 40 \\
\hline Fever & \\
\hline Symptomless & \\
\hline
\end{tabular}

B: bilateral; L: left; R: right. 


\begin{tabular}{|l|c|}
\hline \multicolumn{2}{|l|}{ Table2. Perioperative and operative outcome (n=100) } \\
\hline Operative time (min) & $30.8 \pm 8.78(15-60)$ \\
\hline Volume of perfusate (ml) & $442.7 \pm 109.72(200-1000)$ \\
\hline Laser time (sec) & $370.09 \pm 51.98$ \\
\hline Laser frequency (Hz) & $32.21 \pm 4.24(30-40)$ \\
\hline Laser power (W) & $25.25 \pm 4.60(18-32)$ \\
\hline Duration of hospitalization (d) & $3.30 \pm 0.84$ \\
\hline Complication rate & $69(69 \%)$ \\
\hline Stone-free rate & $89(89 \%)$ \\
\hline
\end{tabular}

Data are mean \pm standard deviation (range).

\begin{tabular}{|c|c|c|c|c|}
\hline Risk factors & $\begin{array}{c}\text { Univariate OR } \\
(95 \% \mathrm{CI})\end{array}$ & & $\begin{array}{c}\text { Multivariate OR } \\
\text { (95\% CI) }\end{array}$ & $\mathbf{p}$ \\
\hline Age & $1.010(0.860 \sim 1.185)$ & 0.907 & & \\
\hline Gender & $2.538(0.705 \sim 9.135)$ & 0.154 & & \\
\hline Stone diameter $(\mathrm{cm})$ & 4.178 (1.156 11.292) & 0.005 & $3.274(1.124 \sim 9.533)$ & 0.030 \\
\hline Stone location & $1.051(0.502 \sim 2.198)$ & 0.896 & $y$ & \\
\hline Staghorn calculi & 24.857 (3.855 160.296) & 0.001 & 13.193 (1.881 92.537) & 0.009 \\
\hline Hydronephrosis & $0.856(0.290 \sim 2.527)$ & 0.779 & & \\
\hline
\end{tabular}

CI: confidence interval; OR: odds ratio. 